\title{
Germanica
}

\section{Thomas Bernhard, Arthur Schopenhauer pour en finir avec la peinture-mimésis}

Thomas Bernhard und Arthur Schopenhauer

Jacques Le Rider

\section{OpenEdition}

\section{Journals}

Édition électronique

URL : http://journals.openedition.org/germanica/2411

DOI : 10.4000/germanica.2411

ISSN : 2107-0784

Éditeur

Université de Lille

Édition imprimée

Date de publication : 30 juin 2000

Pagination : 87-99

ISBN : 9782913857032

ISSN : 0984-2632

\section{Référence électronique}

Jacques Le Rider, "Thomas Bernhard, Arthur Schopenhauer pour en finir avec la peinture-mimésis », Germanica [En ligne], 26 | 2000, mis en ligne le 07 mars 2014, consulté le 06 octobre 2020. URL http://journals.openedition.org/germanica/2411 ; DOI : https://doi.org/10.4000/germanica.2411

Ce document a été généré automatiquement le 6 octobre 2020.

(ㄷ) Tous droits réservés 


\section{Thomas Bernhard, Arthur Schopenhauer pour en finir avec la peinture-mimésis}

Thomas Bernhard und Arthur Schopenhauer

Jacques Le Rider

\section{L'impossibilité de peindre le monde : Gel}

1 Dans le premier grand roman de Thomas Bernhard, Gel (Frost), le peintre Strauch rumine l'impossibilité radicale de peindre le monde ${ }^{1}$. Si Bernhard récuse toute forme de «Ut pictura poesis", c'est parce qu'il conteste, avec une radicalité véritablement gnostique, que le réel soit digne d'être décrit ou dépeint. L'univers créé par le démiurge imposteur est non seulement mal fait, mais malfaisant. L'existence humaine n'a de sens dans ce monde que comme une révolte contre la réalité. La pensée n'a d'autre raison d'être que d'exister contre les faits. «L'homme ne vient pas au monde en ayant pour tâche d'en donner une représentation au moyen de son langage ou de ses systèmes symboliques [...]. Il lui appartient plutôt de se construire une demeure, un centre géométrique qui lui permettre de survivre [...] dans un monde qui ne lui est pas destiné, un monde où il est jeté sans savoir pourquoi ni comment ${ }^{2} »$. Si l'écriture de Thomas Bernhard opère une déconstruction de la notion même de description, c'est pour tirer les conséquences de la haine de la prétendue "réalité », qu'il s'agit justement de déréaliser.

2 Les postmodernes Wenders, Strauss, Handke, pour leur part, ne se sont pas affranchis de la nostalgie des images. Pour Thomas Bernhard au contraire, la cécité serait une forme de bonheur : comme dit Perturbation, «son but est atteint quand il n'y a plus d'image dans son cerveau. Quand, dans le cerveau, les possibilités de représentation sont épuisées ${ }^{3}$ ». Ce n'est plus l'aveuglement qui est une infirmité, mais l'illusion de la clarté; comme dit Wittgenstein dans De la certitude: "Que je comprenne, est-ce 
seulement de la cécité à l'égard de mon propre manque de compréhension? C'est souvent ce qu'il me semble ${ }^{4}$ ».

3 Le monde de Thomas Bernhard est plongé dans les ténèbres: fonds de vallée alpins pluvieux, froids et enveloppés de brouillard; sous-bois pourrissants de sapins et de mélèzes plongés dans une une éternelle pénombre. La clarté qui dissipe ces ténèbres est toute intellectuelle et mortifère: "Nous sommes terrifiés par la clarté qui constitue soudain notre monde, notre monde scientifique; nous gelons dans cette clarté ; mais nous avons voulu ce froid, nous l'avons suscité, nous ne devons donc pas nous plaindre $\mathrm{du}$ froid qui règne maintenant. Le froid augmente avec la clarté. Désormais règneront cette clarté et ce froid ${ }^{5}$ ».

Lorsque la couleur apparait dans un texte de Thomas Bernhard, c'est pour évoquer la vie, les illusions perdues sur le bonheur et sur l'amour. Chantal Thomas consacre un chapitre de son livre sur Bernhard à la " rose jaune ", qu'elle rapproche de manière très suggestive d'un court texte de Borgès, La Rose jaune, qui raconte les derniers instants d'un poète allongé dans son lit de mourant et parcourant du regard les rayons de la bibliothèque où sont alignés ses propres livres. Dans Corrections de Thomas Bernhard, on retrouve dans le tiroir de Roithamer un objet en papier qui n'est ni un manuscrit, ni un imprimé, mais une rose en papier de couleur jaune, souvenir de kermesse de village au cours de laquelle Roithamer avait surpris l'assistance en tirant à la carabine sur des fleurs attachées sur des cibles et en faisant mouche à chaque coup. Cet épisode et cette relique intime évoquent « le monde extérieur comme désirable, comme source possible de bonheur. La rose de papier jaune qui accompagne Roithamer jusqu'aux derniers instants de son existence est le fantôme du jeune homme conquérant, sensuel, mondain, qu'il s'est interdit d'être ${ }^{6} »$. Depuis Gel, Thomas Bernhard prépare son adieu à la vie. Son univers entre dans une ère de glaciation et de décoloration. Seul lui sied le deuil ténébreux des couleurs.

En ce sens, le peintre Strauch est un artiste sans œuvres ${ }^{7}:$ «Er gestand heute, alle von ihm gemalten Bilder verheizt zu haben ${ }^{8}$ ». « Mein Kopf sieht nichts. Alles ist grau. Und gelb. Dann fließen diese Farben ineinander, und ich sehe nichts mehr als Schmerz ». (F, p. 87) «Ich denke darüber nach, was geschieht, wenn eines Tages alles nur mehr schwarz ist, sagte er. Wenn es keine Farbe mehr gibt außer der schwarzen » (F, p. 116). Lorsque son "observateur » et famulus s'enhardit jusqu'à jeter un regard dans la chambre du peintre, il distingue un tableau, peut-être une toile sauvée du grand autodafé, un paysage sans doute, mais un paysage réduit à des taches informes et indistinctes : «Ich öffnete seine Zimmertür und sah ihn in seine Zeitungen vertieft. Ich sah - denn er saß ja hinter seinem Bett, vor dem Bild, dessen Landschaft ich bis jetzt noch nicht enträtselt habe: ein braunes Bild mit großen schwarzen Flecken, die Häuser, aber auch Bäume sein können» (F, p. 129). Ce peintre travaille comme un aveugle : «Im Gegensatz zu anderen Malern, die in hellen Räumen arbeiten müssen, könnte er nur in völlig abgedunkelten Räumen malen. "Es muß finster sein, dann kann ich malen. Nur in völliger Finsternis. Nicht das geringste Licht darf herein. Aber jetzt male ich ja nicht mehr"» (F, p. 131).

Cette cécité constitue une défense, une protection contre les images de la réalité que le peintre ne supporte plus de voir : «Jeder Gegenstand, den ich sehe, tut mir weh. Jede Farbe, die ich anschauen muß. Jede Erinnerung, die auftaucht, alles, alles. Keiner Sache kann ich mehr auf den Grund schauen, denn das würde mich ja augenblicklich auslöschen oder wahnsinnig machen » (F, p. 284). Témoin épouvanté de ce processus de 
« schize », d'Entzweiung, l'observateur-famulus rapporte au frère médecin du peintre : "Was ist es für eine Krankheit? Ihr Bruder verfinstert sich in dem Maße, in welchem er glaubt daß die Welt sich verfinstere, sich alles um ihn und in ihm verfinstere » $(\mathrm{F}$, p. 305).

7 Les seuls paysages que sache représenter ce peintre sont des paysages oniriques, des images mentales : «Ich habe einen Traum gehabt. Die Landschaft, wo der Traum sich abgewickelt hat, in Sekundenschnelle wahrscheinlich, war bald weiß, bald grün, bald grau, bald tiefschwarz. Nichts hatte die Farbe, die ihm nach menschlichem Ermessen zusteht. Der Himmel beispielsweise war grün, der Schnee war schwarz, die Bäume waren blau... die Wiesen so weiß wie Schnee... Plötzlich bemerkte ich, daß hinter mir alles abgestorben war. Abgestorben, tot. Mein großer Kopf lag in einem toten Land. In Finsternis » (F, p. 36 sq.). Plus loin, le peintre Strauch décrit à nouveau un tableau onirique (F, p. 176).

8 Ce peintre est un Orphée à rebours. Loin d'apporter la vie dans la nature, il répand la mort, la décomposition et la destruction. «Als wir den Lärchenwald entlanggingen [...] sagte er : "Wir haben es hier im Augenblick mit der dämonischen Ruhe zu tun. [...] Noch immer hat die Welt von dieser Ruhe eine ganz primitive Vorstellung. Diese Ruhe habe ich zeitlebens als eine Krankheit der erschöpften Natur empfunden, als fürchterlich aufgerissene Abgründe des Gemüts» (F, p. 146 sq.). « "Sehen Sie !", sagte er. Aber ich sah nichts. » dieser Berg hat in mir die Vorstellung eines riesigen Katafalks arbeiten lassen. Sehen Sie! «Tatsächlich hat der Berg die Umrisse eines riesigen Katafalks » (F, p.165). «Ich betrachte mich als den Schöpfer dieses nachmittaglichen Schauspiels, dieser Tragödie ! Dieser Komödie !» (F, p. 189).

Le point de vue du peintre Strauch peut être interprété comme une application radicale des analyses d'Arthur Schopenhauer dans Le monde comme volonté et comme représentation ${ }^{9}, \mathrm{I}, \S 36$ : «Wir können [die Kunst] daher geradezu bezeichnen als die Betrachtungsart der Dinge, unabhängig vom Satze des Grundes, im Gegensatz der gerade diesem nachgehenden Betrachtung, welche der Weg der Erfahrung und Wissenschaft ist. [...] Die dem Satz vom Grunde nachgehende ist die vernünftige Betrachtungsart, welche im praktischen Leben wie in der Wissenschaft allein gilt und hilft : die vom Inhalt jenes Satzes wegsehende ist die geniale Betrachtungsart, welche in der Kunst allein gilt und hilft. [...] Nur durch die [...] im Objekt ganz aufgehende reine Kontemplation werden Ideen aufgefaßt, und das Wesen des Genius besteht eben in der überwiegenden Fähigkeit $\mathrm{zu}$ solcher Kontemplation: da nun diese ein gänzliches Vergessen der eigenen Person und ihrer Beziehungen verlangt ; so ist Genialität nichts anderes als die vollkommenste Objektivität, [...] entgegengesetzt der subjektiven, auf die eigene Person, d.i. den Willen gehenden » (WWV, p. 265 sq.).

Tout se passe comme si le génie du peintre Strauch s'était manifesté pour faire de cet artiste le miroir de l'essence du monde. Du génie, il a les traits de caractère, que son entourage interprète comme une "perturbation » ou comme de la folie : «Der "geniale Ausdruck" eines Kopfes besteht darin, daß ein entschiedenes Übergewicht des Erkennens über das Wollen darin sichtbar ist, folglich auch ein Erkennen ohne alle Beziehung auf ein Wollen, d.i. reines Erkennen, sich darin ausdrückt» (WWV, p. 269). D'où cette inquiétante propension au monologue ininterrompu, que l'observateurfamulus voit avec terreur se développer sans frein. «Weil ihre Erkenntnis sich zum Teil dem Dienste des Willens entzogen hat, werden sie im Gespräche nicht sowohl an die Person denken, zu der, sondern mehr an die Sache, wovon sie reden, die ihnen lebhaft 
vorschwebt [...]. Daher sind sie zu Monologen geneigt und können überhaupt mehrere Schwächen zeigen, die sich wirklich dem Wahnsinn nähern. Daß Genialität und Wahnsinn eine Seite haben, wo sie aneinander grenzen, ja ineinander übergehn, ist oft bemerkt [...] worden » (WWV, p. 272).

«Wenn [...] ein [...] Kummer, ein [...] schmerzliches Wissen oder Andenken so qualvoll ist, daß es schlechterdings unerträglich fällt und das Individuum ihm unterliegen würde - dann greift die dermaßen geängstigte Natur zum Wahnsinn als zum letzten Rettungsmittel des Lebens. [...] Als Beispiel betrachte man den rasenden Aiax, den König Lear und die Ophelia » (WWV, p. 276). Ce passage de Schopenhauer éclaire un des thèmes récurrents de Thomas Bernhard, la référence au roi Lear, dont il sera encore question un peu plus loin, à propos de Minetti. "Eines Tages habe er aber von den Kunststücken genug gehabt - "von Kunststücken hat man gleich genug" - und sich der reinen wirklichen Kunst, der Kunst, die auf keinen Kunststücken beruht, zugewandt. [...] Eine reine Kunst also vorzutragen, wie sie das Theater darstelle, eine Kunst, eben ohne Kunststücke, sagen wir : "den König Lear zu verkörpern" » (F, p. 238. À cet endroit de Gel, c'est un vagabond rencontré par le peintre Strauch dans le bois de mélèzes qui parle).

La peinture de paysage, qui est le genre favori du peintre Strauch, est considérée par Schopenhauer au $\S 43$ du Monde... comme un stade intermédiaire de l'objectivité de la volonté. La peinture de paysage a au moins le mérite de valoir un peu mieux que l'architecture et le génie hydraulique, degrés les plus inférieurs de l'objectivité de la volonté, à l'instar de l'art des parcs et jardins. "Die Wirkung der eigentlichen Landschaftsmalerei ist nun zwar im ganzen auch noch von dieser Art : allein weil die dargestellten Ideen als höhere Stufen der Objektivität des Willens schon bedeutsamer und vielsagender sind ; so tritt die objektive Seite des ästhetischen Wohgefallens schon mehr hervor und hält der subjektiven das Gleichgewicht. Das reine Erkennen als solches ist nicht mehr ganz die Hauptsache; sondern mit gleicher Macht wirkt die erkannte Idee, die Welt als Vorstellung auf einer bedeutenden Stufe der Objektivation des Willens » (WWV, p. 309). Mais la peinture, dans la hiérarchie des arts, est placée très en dessous de la musique à qui schopenhauer prête le plus grand pouvoir d'« objectivation de la volonté du monde ».

Ce peintre que nous présente Thomas Bernhard parle comme un métaphysicien gnostique, comme un de ces marcionites, dont Schopenhauer dit dans dans les Compléments au Monde... (IV, §48, «De la négation de la volonté de vivre $)^{10}$ : "[Clemens] wirft den Markioniten vor, daß sie nach dem Vorgang des Platon und Pythagoras die Schöpfung schlecht befunden hätten, indem Markion lehre, es sei eine schlechte Natur, aus schlechtem Stoff; daher man diese Welt nicht bevölkern, sondern der Ehe sich enthalten solle. [Clemens] sieht darin ihren schreienden Undank, ihre Feindschaft und Empörung gegen den, dessen Werk sie selbst seien und dennoch von seinen Schöpfungen Gebrauch zu machen verschmäheten » (WWV II, p. 796).

Un mot sur la « lecture bernhardienne » de Schopenhauer. S'il est incontestable que ce philosophe a été une des principales références de Thomas Bernhard (en 1975, il déclarait qu'il avait lu Le Monde comme volonté et comme représentation dès l'âge de 12 ans, " en cachette et volontairement ${ }^{11}$ ), il serait vain d'attendre une quelconque systématicité de la pensée de Thomas Bernhard qui, dans Maîtres anciens, s'est exprimé sans ambages à propos de sa manière de lire les classiques : «Wer alles liest, hat nichts begriffen, sagte er. Es ist nicht notwendig, den ganzen Goethe zu lesen, den ganzen 
Kant, auch nicht notwendig den ganzen Schopenhauer; ein paar Seiten Werther, ein paar Seiten Wahlverwandtschaften und wir wissen am Ende mehr über die beiden Bücher, als wenn wir sie von Anfang zum Ende gelesen hätten, was uns in jedem Fall um das reinste Vergnügen bringt » (AM, p. 40). Pour notre part, nous ne prétendons pas faire le point sur l'ensemble de la question des relations entre Thomas Bernhard et Schopenhauer ${ }^{12}$, mais en éclairer un aspect qui nous semble essentiel : la philosophie de la peinture et de l'image.

\section{Les masques de James Ensor: Minetti}

Minetti n'est pas seulement une pièce dédiée à la réflexion sur l'art dramatique et sur le destin de l'acteur de théâtre. Thomas Bernhard rend aussi un hommage d'une rare ferveur au peintre James Ensor (1860-1949), cet individualiste inclassable qui fut un des maitres du post-impressionnisme avant de s'affirmer comme un des plus grands peintres expressionnistes. Dans cette période expressionniste, Ensor sut renouer avec la grande tradition du réalisme critique et grinçant des anciens Flamands, de Bosch et de Brueghel. Ses thèmes sont, à y regarder de près, fort «bernhardiens » : la souffrance de l'individu génial mis au ban d'une société mesquine et empoisonnée, l'entrée du Christ à Jérusalem, sous la huée et la risée qui annoncent les persécutions, l'artiste comme figure moderne de l'ecce homo sans phrase et sans grandeur, mais au contraire misérable et pitoyable, l'existence comme "maladie à la mort » selon la formule de Kierkegaard, les masques et les squelettes, macabres et grotesques, terrifiants et dérisoires, princes métaphysiques et petits-bourgeois caricaturaux.

Il y a beaucoup de points communs entre Bernhard et Ensor, dont la vie "s'est écoulée, à Ostende, presque tout entière. Il y a subi l'interminable et ensevelissant ennui de la province qui tombe sur l'âme comme une poussière sur le corps; il y a connu la moquerie et la haine ; le potin et la risée ; il y a rencontré les contrariétés domestiques, l'incompréhension inévitable, la déréliction. les heures noires lui ont fait cortège au long des jours gris ${ }^{13}$.» Comme le grand «exagérateur » (Übertreibungstler) Thomas Bernhard qui se confondait acec ses personnages avec son peintre Strauch, avec Reger, James Ensor avait fini par se transformer lui-même en un de ses masques, peut-être ignorait-il lui-même en un de ses masques : « lorsqu'Ensor introduisit en sa peinture un tel peuple étrange et tragique de masques, peut-être ignorait-il lui-même qu'à certain moment ils lui fausseraient à tel point la notion du réel qu'il ne verrait plus qu'eux de vraiment vivants sous le soleil et qu'un jour il prendrait place parmi leur multitude comme s'il était lui-même quelqu'un de leur lignée et de leur race ${ }^{14}$. "

Plus nettement que dans Gel, le roi Lear est le personnage clef, qui fait le lien entre Minetti et James Ensor :

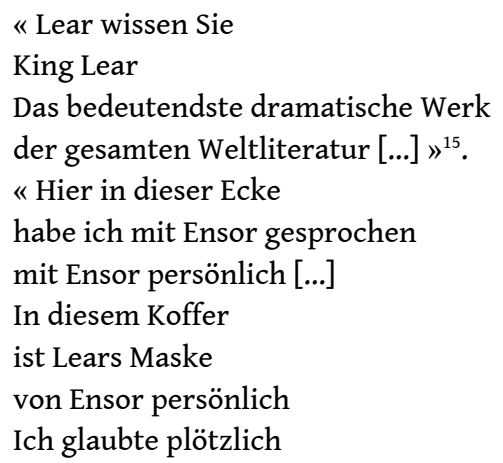


er sei Shakespeare

während ich doch mit Ensor gesprochen habe [...]» (M, p. 553). qui les directeurs de théâtre ne confient plus aucun rôle, et dont le seul rôle, si important qu'il suffit à manifester tout son génie d'acteur, consiste à se jouer lui-même, à jouer le rôle de l'artiste dramatique en attente, dans les salles de réception d'un hôtel d'Ostende, un soir de Saint-Sylvestre. Cet acteur génial qui ne joue que son propre destin parvient mieux qu'aucun autre à «l'objectivation de la volonté ». Par là, il rejoint le peintre Ensor, il partage avec lui l'angoisse qui caractérise l'artiste profond ( Ich habe Angst/Die Künstler haben alle Angst », M, p. 556).

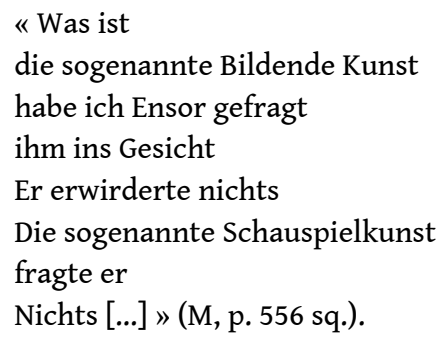

Ce soir-là, tous les invités, les visiteurs, les voyageurs logés à l'hôtel s'apprêtent à faire la fête et mettent un masque ( Die Leute retten sich/durch diesen Tag/indem sie sich betrinken/Masken aufsetzen », $M$, p. 588). Ce qui ramène le monologue de l'acteur désœuvré à James Ensor :

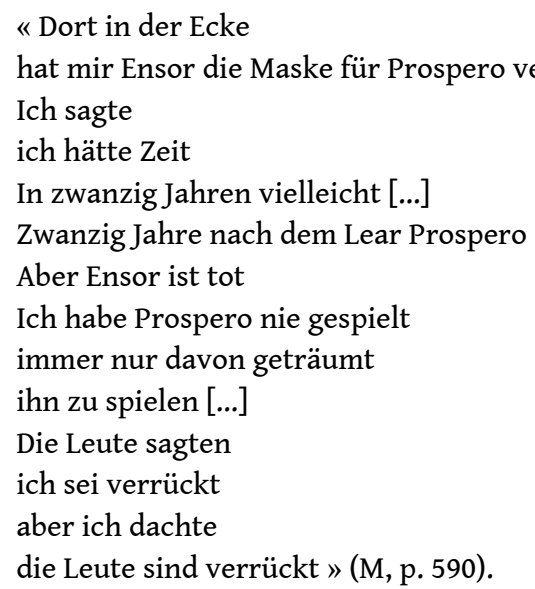

Arthur Schopenhauer, avant Thomas Bernhard, l'avait écrit : « Meine eigene vieljährige Erfahrung hat mich auf die Vermutung geführt, daß Wahnsinn verhältnismäßig am häufigsten bei Schauspielern eintritt» (WWV II, p.515; III, §32, «Über den Wahnsinn »). Dans Le monde comme volonté et comme représentation, la signification de l'art du tragédien est définie en ces termes : «Der wahre Sinn des Trauerspiels ist die tiefere Einsicht, daß, was der Held abbüßt, nicht seine Partikularsünden sind, sondern die Erbsünde, d.h. die Schuld des Daseins selbst: [...] «Da die größte Schuld des Menschen/Ist, daß er geboren ward «(Das Leben ein Traum, I, 2), wie Calderon es geradezu ausspricht » (WWV, § 51, p. 353).

\section{Extinction de la peinture et des couleurs}

Ce roman de 1986, l'un des plus grands de Thomas Bernhard, placé sous l'exergue de Montaigne : «Je sens la mort qui me pince continuellement la gorge ou les reins $[. ..] »^{16}$ 
accorde une importance considérable à Schopenhauer : "Plötzlich habe ich Gambetti einen Schopenhauerschen Satz aus der Welt als Wille und Vorstellung zuerst auf Deutsch, dann auf Italienisch vorgesprochen [...]» (A, p. 9). Alors que les ancêtres du narrateur ont accumulé à Wolfsegg une bibliothèque considérable, ses parents n'ont pas ajouté un seul volume à ces collections : «Mein Vater las kein Buch, meine Mutter blätterte nur $\mathrm{ab}$ und zu in alten naturwissenschaftlichen Büchern, um sich an den farbenprächtigen Stichen, die diese Bücher schmücken, zu ergötzen » (A, p. 23). Le plaisir des couleurs est donc l'apanage du mauvais goût béotien. Les esprits supérieurs ne supportent en vérité aucune image de la réalité. "Das Fotografieren ist eine gemeine Sucht, von welcher nach und nach die ganze Menschheit erfaßt ist, weil sie in die Verzerrung und die Perversität nicht nur verliebt, sondern vernarrt ist und tatsächlich vor lauter Fotografieren mit der Zeit die verzerrte und die perverse Welt für die einzig wahre nimmt » (A, p. 29). La seule couleur que supporte le narrateur est à présent, affirme-t-il, le vert bouteille foncé (" grünschwarz, meine Lieblingsfarbe », A, p. 624), une nuance de cette couleur verte dans laquelle s'habillent, nous a-t-il dit, les Autrichiens, les chasseurs et les nazis, ce qui revient au même, soutient-il, puisque les Autrichiens sont tous des chasseurs et tous des nazis.

Le seul cadre de vie qui convienne à cet iconoclaste radical sera décoloré et sans image. « Ich kenne kein schöneres Vorhaus, hatte ich zu Gambetti gesagt, [...] an den Wänden ist nicht der geringste Schmuck, kein bild, nichts. Die Wände sind weißgekalkt und wirken unerbittlich auf den Betrachter » (A, p. 169). Dans les chambres des enfants, à Wolfsegg, « waren alle Räume, also auch alle Zimmer weiß gekalkt. Die Vorhänge waren dunkelgrün, fast schwarz in den Zweiterstockzimmern, dunkelrot, fast schwarz in den Ersterstockzimmern » (A, p. 178). Même les tableaux accrochés aux murs de Wolfsegg, dont le narrateur se dit à présent qu'ils étaient peut-être des pièces de musée, se réduisaient à "mehr oder weniger verdüsterte Farbflecken » (A, p. 361), que personne ne songeait plus à regarder. Quand il revient dans la Villa des enfants, le narrateur n'aperçoit plus aucun des tableaux qu'il gardait en mémoire: «Die Mutter habe die Bilder, die von frühen Vorfahren gemalt worden sind, an einen Antiquitätenhändler in Wels verkauft, wie ich gleich feststellte, verschleudert » (A, p. 460). Toutes les couleurs et les images associées au bonheur de l'enfance ont fait l'objet d'une extinction férocement méthodique. Il ne reste plus dans la réalité que les stucs blancs et incolores des plafonds: "An den Decken sind genau die Stukkaturen angebracht, die ich Gambetti zu beschreiben versucht habe, lauter Szenen aus klassischen Schauspielen wie beispielsweise aus dem Nathan von Lessing oder den Räubern oder aus dem Urfaust » (A, p. 462).

\section{Assez de ces Maîtres anciens !}

De la peinture, il est beaucoup question dans Maitres anciens. Mais cette discipline artistique fait l'objet d'un constat sans appel qui récapitule très exactement la hiérarchie schopenhauérienne des arts: "Von der Malerei sei er schon sehr früh enttäuscht gewesen, sie war ihm von Anfang an die ungesittige unter den Künsten. Es las viel und leidenschaftlich, aber auf die Idee, selbst zu schreiben, sei er niemals gekommen, er hatte es sich nie zugetraut. Die Musik liebte er von Anfang an, in der Musik fand er schließlich zusätzlich, was er in der Malerei wie auch in der Literatur vermißte ${ }^{17}$.» 
24 Reger, dont Maîtres anciens rapporte les monologues et les points de vue, est « ein wissenschaftliches Genie, ja sogar ein menschliches Genie ", dont la «Geistesproduktionsstätte » (AM, p. 25) est le Kunsthistorisches Museum de Vienne. Reger est essentiellement musicologue, mais à son avis «Die sogenannte Bildende Kunst ist für einen Musikwissenschaftler, wie ich einer bin, von höchster Nützlichkeit [...], umgekehrt denke ich, daß es für einen Maler beispielsweise von größtem Vorteil ist, wenn er sich der Musik widmet. » (AM, p. 36). Ce roman (cette " comédie ») opère à nouveau la synthèse des arts et de la philosophie. Dans Gel, le peintre se révélait un philosophe schopenhauérien. Dans Minetti, la confrontation entre James Ensor et le tragédien voué à jouer le roi Lear se déroulait comme un dialogue entre un pessimiste et un fou, tous deux attachés à " objectiver la volonté du monde ». Dans Maîtres anciens, le musicologue présente une métaphysique de l'art fort proche de celle d'Arthur Schopenhauer. «Ich bin Maler und Musiker und Schriftsteller in einem » (AM, p. 107). Ce maître du jugement esthétique est aussi un maître-lecteur des philosophes et des auteurs classiques: "Meine Art $\mathrm{zu}$ lesen ist die eines hochgradig talentierten Umblätterers » (AM, p. 11).

L'idéal de Reger est à l'opposé du classicisme winckelmannien : «Das Ganze und das Vollkommene ist uns unerträglich, sagte er » (AM, p. 41). Regarder un tableau, lire un texte, écouter une composition musicale ne consistent plus à «se plonger dans une œuvre » selon l'attitude herméneutique, mais à entreprendre, avec l'agressivité qu'exige l'objectivité, la déconstruction de l'œuvre ${ }^{18}$ : «Wie müssen Bach hören und hören, wie er scheitert [...] Wir lieben ja Pascal nicht, weil er so vollkommen ist, sondern weil er im Grunde so hilflos ist [...] Nur die Bücher lieben wir in Wahrheit, die kein Ganzes, die chaotisch, die hilflos sind » (AM, p. 43). «Ja, sagte er, El Greco, schön, aber der gute Mann hat keine Hand malen können» (AM, p.44). Finalement tout sombre dans la dérision, l'amateur d'art «verdirbt sich alles und jedes, selbst das Geliebteste » (AM, p. 68).

Thomas Bernhard lui-même souhaitait-il «être aimé » de cette manière-là ? Aimé au point qu'on finirait par le trouver grotesque et repoussant? Aimé comme on aime ces grands ratés de l'histoire de l'art, de la littérature et de la philosophie: Stifter, Heidegger et Bruckner? Reger ne conteste pas qu'il se sente quelque affinités avec ces créateurs insupportables : "Ich bin mit Stifter verwandt und mit Heidegger und mit Bruckner und mit einem Doppelmörder, der in Steyr und Stein seine Strafe abgebüßt hat, habe ich sehr oft gesagt, auch wenn man mich nicht danach gefragt hat, sagte Reger gestern » (AM, p. 100).

27 Grandes ne sont que les œuvres qui peuvent se laisser déconstruire. Les classiques ne s'imposent pas en raison de leur monumentalité, mais en raison de leur fragilité et de leur ambivalence. Ils n'appellent pas l'admiration, mais l'intérêt, l'estime, la « prise en compte ». " Ich war ja zeitlebens immer weit davon entfernt, ein Bewunderer zu sein, Bewunderung ist mir fremd [...]. Der eigentliche Verstand kennt die Bewunderung nicht, er nimmt zur Kenntnis, er respektiert, er achtet, das ist alles, sagte er » (AM, p. 122). Les célébrités (les «Berühmten », écrit Bernhard qui a intitulé ainsi une de ses pièces de théâtre) de la culture nationale (Mahler, Schiele, etc.) sont " enervierend» (AM, p. 181), à la fois au sens d'irritants et de débilitants. "Wien ist ein Kulturbegriff, sagte Reger, auch wenn in Wien schon lange fast keine Kultur mehr ist » (AM, p. 183).

$28 \mathrm{Au}$ terme de cette liquidation en règle de tout le patrimoine artistique et intellectuel autrichien et européen, Thomas Bernhard pousse à son comble l'esthétisme. « Ich bin 
in die Kunst hineingeschlüpft, um dem Leben zu entkommen» (AM, p. 190). «Zu den Alten Meistern muß ich gehen, um weiter existieren zu können, genau zu diesen sogenannten Alten Meistern, die mir ja längst und schon seit Jahrzehnten verhaßt sind» (AM, p. 208). «Die Musik rettet mich ja immer wieder» (AM, p. 243). Nouveau des Esseintes, Reger a pris en horrreur tout ce qui serait nature ou, de près ou de loin, naturel. Même la lumière ne lui est plus supportable que comme éclairage artificiel: «Er geht der Sonne aus dem Weg, nichts flieht er so, wie die Sonne. Ich hasse die Sonne, Sie wissen, ich hasse die Sonne wie nichts sonst auf der Welt, sagt er " (AM, p. 101). Mais la fuite hors de la vie ne donne pas accès au paradis artificiel de l'art. L'esthétisme poussé à l'extrême de Thomas Bernhard et de son personnage Reger ne conduit qu'au nihilisme le plus radical, à une sorte de suicide esthétique. Car rien n'a plus de valeur ni de consistance face à l'absurdité de l'existence et de la mort. L'amateur d'art s'expose à une inévitable déception : «Wir haben schließlich überhaupt kein Vergnügen mehr an der Kunst » (AM, p. 227). Le bilan de Maîtres anciens est plus que maigre: il n'en reste qu'un paysage ravagé par le souffle d'une véhémence désespérée qui jette ses imprécations à la face de la Volonté du monde, de la vie et de la culture.

Alles Gesagte stellt sich über kurz oder lang als Unsinn heraus, aber wenn wir es überzeugend sagen, mit der unglaublichsten Vehemenz, die uns möglich ist, ist es ja kein Verbrechen, sagte er (AM, p. 187).

\section{NOTES}

1. Cf. J. Le Rider, Les couleurs et les mots, Paris, Presses Universitaires de France, coll. Perspectives critiques, 1997, 21999.

2. Aldo G. Gargani, La phrase infinie de Thomas Bernhard, trad. Jean-Pierre Cometti, Éditions de l'éclat, coll. « Tiré à part », 1990, p. 11.

3. Thomas Bernhard: Perturbation, trad. B. Kreiss, Paris, Gallimard, coll. L'imaginaire, 1989, p. 209 ; Verstörung, Frankfurt/Main, Suhrkamp, 1986, p. 185.

4. Ludwig Wittgenstein Über Gewißheit, Oxford, 1964, § 418 ; De la certitude, trad. J. Fauve, Paris Gallimard, 1976, p. 103. 1976, p. 103.

5. Thomas Bernhard, extrait du discours de remerciement pour le prix de littérature de Brême, prononcé en 1965, in Thomas Bernhard, Ténèbres, éd. par Claude Porcell, Paris, éditions Maurice Nadeau, 1986, p. 31.

6. Chantal Thomas, Thomas Bernhard, Paris, Le Seuil, coll. Les contemporains, 1990, p. 160.

7. Wendelin Schmidt-Dengler fait remarquer qu'on pourrait prendre ce prétendu "peintre » pour un "créateur de mots", pour un artiste du verbe plutôt que du pinceau. W. SchmidtDengler, "Zurück zum Text: Vorschläge für die Lektüre von Thomas Bernhards Frost», in Thomas Bernhard. Beiträge zur Fiktion der Postmoderne. Londoner Symposion, éd. par W. SchmidtDengler, A. Stevens, F. Wagner, Frankfurt/Main, etc., Peter Lang, 1997 (p. 201-220), p. 209. Toutefois nous nous efforcerons de prendre le texte de Bernhard au pied de la lettre et de considérer ce peintre bel et bien comme un peintre. 
8. Thomas Bernhard, Frost, Frankfurt/Main, Suhrkamp Taschenbuch $n^{\circ} 47,1976$, p. 33 (Les citations extraites de cet ouvrage seront mentionnées dans la suite du texte avec la référence abrégée «F, p. »).

9. Citations d'après Arthur Schopenhauer, Sämtliche Werke, éd. par Wolfgang Frhr. von Löhneysen, Darmstadt, Wissenschaftliche Buchgesellschaft, 1974, vol. I, Die Welt als Wille und Vorstellung, abrégé « WWV ».

10. Citations d'après Arthur Schopenhauer, Sämtliche Werke, éd. par Wolfgang Frhr. von Löhneysen, Darmstadt, Wissenschaftliche Buchgesellschaft, 1974, vol. II, Die Welt als Wille und Vorstellung, abrégé « WWV II ».

11. Jens Dittmar, Thomas Bernhard. Werkgeschichte, Frankfurt/Main, Suhrkamp, $2^{\mathrm{e}}$ édition, 1990, p. 164.

12. Gerald Jurdzinski, Leiden an der « Natur». Thomas Bernhards metaphysische Weltdeutung im Spiegel der Philosophie Schopenhauers, Frankfurt/Main, etc., Peter Lang, 1984.

13. Émile Verhaeren, Sur James Ensor, Bruxelles, Éditions Complexe, coll. «Le regard littéraire», 1990, p. 121.

14. Ibid., p. 86.

15. Minetti, in Thomas Bernhard, Die Stücke, Frankfurt/Main, Suhrkamp, 1983, p. 548 (dans la suite, les citations de Minetti sont faites d'après cette édition, référence abrégée « $\mathrm{M}$ »).

16. Épigraphe de Thomas Bernhard, Auslöschung. Ein Zerfall, 1986, cité dans la suite du texte d'après l'édition Frankfurt/Main, Suhrkamp, 1988 (Suhrkamp Taschenbuch; 1563), référence abrégée « A » (Thomas Bernhard, Extinction. Un effondrement, trad. Gilberte Lambrichs, Gallimard, 1990).

17. Thomas Bernhard, Alte Meister. Komödie, 1985, citée d'après l'édition Suhrkamp Taschenbuch $\mathrm{n}^{\circ} 1553,1988$, p. 104, dans la suite référence abrégée « $\mathrm{AM}$ » (Thomas Bernhard, Maîtres anciens. Comédie, trad. Gilbert Lambrichs, Paris, Gallimard, 1988).

18. Wendelin Schmidt-Dengler, «Liquidation durch Anschauung. Zur Kunstvernichtung in den Alten Meistern ", in W. Schmidt-Dengler, Der Übertreibungskünstler. Zu Thomas Bernhard, Wien, Sonderzahl, 1986, S. 87-92; Vgl. Ingeborg Hoesterey, "Visual Art as Narrative Structure Thomas Bernhard's Alte Meister », in Modern Austrian Literature, vol. 21, Nr. 314, 1988, S. 117-122.

\section{RÉSUMÉS}

La présence de Schopenhauer chez Thomas Bernhard a souvent été envisagée dans le contexte de réflexions sur le "pessimisme» de Bernhard. Dans la présente étude, c'est la philosophie schopenhauérienne de l'art qui est plus particulièrement mise en relation avec quelques œuvres de Thomas Bernhard. Dans le roman Gel, le peintre philosophant Strauch expose une conception de la peinture qui s'accorde parfaitement avec la hiérarchie schopenhauérienne des disciplines artistiques : la peinture est très nettement inférieure à la musique si l'on considère son " pouvoir d'objectivation de la volonté du monde ». La pièce Minetti rend hommage au peintre James Ensor tout en développant une philosophie de la tragédie et une phénoménologie existentielle de l'acteur qui se révèlent très proches de celles de Schopenhauer. Dans le roman Extinction, la tendance « iconoclaste » de Thomas Bernhard s'accentue. C'est dans le roman Maîtres anciens que le verdict final sur la peinture est rendu, en termes parfaitement conformes à Schopenhauer : 
toutes les collections du Kunsthistorisches Museum de Vienne ne valent pas grand-chose et comptent moins que la littérature et surtout que la musique.

Thomas Bernhards Schopenhauer-Rezeption wurde bisher meistens im Kontext von Überlegungen zu Bernhards «Pessimismus» in Betracht gezogen. In der vorliegenden Studie wird vor allem Schopenhauers Philosophie der Künste in Verbindung mit einigen zentralen Werken Thomas Bernhards gesetzt. Im Roman Frost trägt der philosophierende Maler Strauch eine Auffassung von der Malerei vor, die mit der Schopenhauerschen Rangordnung der Künste vollkommen übereinstimmt: Die Malerei sei der Musik eindeutig unterlegen, wenn man die Künste nach deren Vermögen zur «Objektivation der Welt als Willens » einstuft. Das Stück Minetti enthält eine Hommage an den Maler James Ensor und entwirft zugleich eine Philosophie der Tragödie und eine Existential-Phänomenologie des Schauspielers, die in unmittelbarer Nähe zu Schopenhauer stehen. Im Roman Auslöschung fällt das endgültige Urteil über die Malerei im Sinne Schopenhauers : Alle Bilder der Sammlungen des Wiener Kunsthistorischen Museums sind nicht viel wert und zählen weniger als Literatur und vor allem als Musik.

\section{AUTEUR}

JACQUES LE RIDER

École pratique des Hautes Études,

Section des Sciences historiques et philologiques 